\title{
Impressum, Vol. 3, No. 3, 1980
}

\section{Herausgeber}

Prof. Dr. S. Eckhardt, Staatliches Onkologisches Institut, Budapest

Prof. Dr. R. Gross, Medizinische Universitätsklinik, Köln

Prof. Dr. J. H. Holzner, Institut für pathologische Anatomie,

Universität Wien

Prof. Dr. K. Munk, Deutsches Krebsforschungszentrum, Institut für

Virusforschung, Heidelberg

Prof. Dr. G. P. Murphy, Roswell Park Memorial Institute,

Buffalo (N.Y.)

Prof. Dr. A. Stacher, Hanusch-Krankenhaus, Wien

Prof. Dr. Dr. St. Tanneberger, Zentralinstitut für Krebsforschung,

Akademie der Wissenschaften, Berlin/DDR

Prof. Dr. W. Wilmanns, Medizinische Klinik III, Klinikum Groß-

hadern, Universität München

Prof. Dr. Dr. H. Wrba, Institut für Krebsforschung, Universität Wien

Wissenschaftlicher Beirat:

H. W. Bauer, München; H. Denck, Wien; V. Diehl, Hannover; F. Douwes, Göttingen; P. Drings, Heidelberg; H. Ehrhart, München; J. Fischer, Mainz; W. M. Gallmeier, Nürnberg; H. Gerhartz, Berlin; E. H. Graul, Marburg/Lahn; E. Hauptmann, Zagreb; H. Heimpel, Ulm; K. P. Hellriegel, Köln; Ch. Herfarth, Ulm; R. Hünig, Basel; W. Hunstein, Heidelberg; H. O. Klein, Köln; H. Löffler, Lahn Gießen; U. Mohr, Hannover; F.-L. Müller, Bonn; G. Nagel, Göttingen; A. Pfleiderer, Freiburg; Mildred Scheel, Bonn; E. Scherer, Essen; S. Seeber, Essen; H. J. Senn, St. Gallen; W. Vahlensieck, Bonn; H. D. Waller, Tubingen; M. Wannenmacher, Freiburg; K. Wilms, Tubingen; K. zum Winkel, Heidelberg

Die Zeitschrift erscheint zweimonatlich; pro Jahr erscheint 1 Band zu je 6 Heften.

Bezugspreis für Jahrgang 3, 1980 DM 104,-/ÖS 728,-/SFr. 104,-. 1 Einzelheft kostet DM 22,/OS 154,-/SFr. 22,-, einschließlich MwSt., zuzüglich Postgebühren.

Der Abonnementspreis ist im voraus zahlbar. Die Lieferung der Zeitschrift läuft weiter, wenn sie nicht spätestens 4 Wochen vor Abschluß eines Bandes abbestellt wird.

Abonnementsbestellungen können bei jeder Buchhandlung, bei der Post oder direkt beim Verlag aufgegeben werden: Bundesrepublik Deutschland/Österreich: S. Karger GmbH, Postfach 2, D8034 Germering/München, Tel: (089) 844021, Telex: 524865 D, Postscheckkonto: München 400 80-807 Schweiz: S. Karger AG, Allschwilerstr. 10, Postfach, CH-4009 Basel, Tel.: (061) 390880, Telex62652CH

Anzeigen

S. Karger Verlag für Medizin und Naturwissenschaften $\mathrm{GmbH}$, Postfach 2, 8034 Germering, Telefon (089) 844021.

Offizielles Organ der Deutschen Gesellschaft für Hämatologie und Onkologie 
und der österreichischen Gesellschaft für Hämatologie und Onkologie

Satz und Druck: Buch- und Offsetdruckerei Walter Biering KG, 8000 München 40

Für den Inhalt außerhalb des redaktionellen Teiles (insbesondere Anzeigen,

Industrieinformationen, Pressezitate und Kongreßinfor-mationen) übernehmen Herausgeber und Veflag keine Gewähr.

Alle Rechte, insbesondere das Recht der Vervielfältigung und Mikro-kopie sowie der

Übersetzung, vorbehalten. Nachdruck, auch auszugs-weise, nur mit Genehmigung des Verlages.

(C) Copyright 1980 by S. Karger Verlag für Medizin und Naturwissen-schaften GmbH, Postfach

2, D-8034 Germering

Sonderhefte bzw. Sonderbände sind nur in beschränkter Auflage vor-rätig. Der Preis ist abhängig vom Umfang. Abonnenten erhalten diese zu einem um 20 \% vom Ladenpreis ermäßigten

Vorzugspreis.

Beiträge zur Onkologie

Sonderband 1: Chemotherapie urologischer Malignome

Prostata, Harnblase, Penis/Harnröhre. Seminar des Berufsverbandes

Deutscher Urologen e. V., Mainz, März 1979

190 S., 70 Abb., 59 Tab., brosch., DM 35,-

Beiträge zur Urologie

Sonderband 1: Diagnostik und Therapie des Prostatakarzinoms Symposium der Urologischen Universitätsklinik München in Zusam-menarbeit mit dem Tumorzentrum München, München, November '78 168 S., 67 Abb., 48 Tab., brosch., DM 40,-

96

Hinweise für Autoren

Bitte senden Sie Ihr Manuskript direkt an den Verlag S. Karger.

Es werden nur Originalarbeiten in deutscher Sprache veröffentlicht.

Das Manuskript soil in druckreifem .Zustand in dreifacher Ausfertigung eingereicht werden. Die Entscheidung über die Aufnahme erfolgt aufgrund mehrerer Gutachten. Eine Kopie des Manuskripts soil grundsätzlich beim Autor verbleiben.

Die typographische Gestaltung und redaktionelle Bearbeitung ist dem Verlag vorbehalten.

Der Verlag erwirbt alle Rechte an Sonder- und Abdrucken in anderen Publikationen. Autorkorrekturen über $10 \%$ der Satzkosten hat der Autor zu tragen. Dem Autor stehen 40 Gratissonderdrucke seiner Arbeit zu.

Markennamen müssen, wenn sie geschützt sind, mit einem hochge-stellten ${ }^{\circledR}$ bezeichnet werden. Die Verantwortung für die korrekte Befolgung dieser Vorschrift liegt ausschließlich beim Autor. Abkiirzungen sollen möglichst sparsam verwendet werden. Bei spe-ziellen Abkürzungen sollte der Autor jeweils beim ersten Auftreten das Wort ausschreiben und die später verwendete Abkürzung in Klam-mern folgen lassen: z.B. Adenosin-monophosphat (AMP).

Institutsangabe in der Sprache der Arbeit.

Zusammenfassungen sollen 20 Schreibmaschinenzeilen nicht über-schreiten. Eine Fassung in englischer Sprache und der englische Titel der Arbeit müssen mitgeliefert werden, auch wenn die Arbeit in deutscher Sprache abgefaßt ist. In die Zusammenfassung gehören keine Literaturzitate, Tabellen, Abbildungen und Abbildungshinweise sowie allgemein gehaltene Aussagen. 
Schliisselwörter sollen ebenfalls in deutscher und englischer Sprache abgefaßt werden. Zu einer Arbeit gehören drei bis fünf Key Words.

Tabellen, Abbildungen und Literaturzitate müssen im Text erwähnt werden.

Abbildungsvorlagen dürfen nicht aufgeklebt, gelocht oder mit Büro-klammern angeheftet eingereicht werden und sollen auf der Rückseite den Namen des Autors, den Titel des Beitrags sowie die Bildnummer tragen.

Adresse des Autors ist am Schluß der Arbeit vollständig anzugeben.

Literaturverzeichnis. Es soil nur die im Text zitierten Publikationen, diese dafür lückenlos, enthalten. Die Literatur ist entweder alpha-betisch zu ordnen oder entsprechend der Reihenfolge im Text zu numerieren.

Zur Therapie maligner Tumoren:

WOBE-MUGOS

- WOBE-MUGOS $\AA^{-}$- Gemisch proteolytischer Enzyme - lost nekrotische und mitotisch entartete Zellenauf, ohne gesunde Zellen zu beeinflussen.

- WOBE-MUGOS ${ }^{\circledR}$ wirkt fibrinolytisch und virostatisch.

Vorzüge: Im Gegensatz zu den klassischen Cytostatika und zu ionisierenden Strahlen besitzt WOBE-MUGOS $®$

keinerlei nachteilige Wirkung auf das Knochenmark und den Immunapparat.

Indikatione $\prod$ : Langzeitbehandlung aller malignen Tumoren $\cdot$ Zusatzbehandlung während der $\mathrm{m}$

Strahlen-, Immun- und Chemotherapie·Metastasen- und Rezidivprophylaxe

-Palliativbehandlung von Tumorkranken im Endstadium·zur intracavitären und intratumoralen Injektion'zur Präventiv-Behandlung des Krebses

Zusammersetzung: $100 \mathrm{mg}$ Enzymgemisch enthalten proteolytische Enzyme aus fraktio $\pi$ ierten Hydrolysaten: Rinderpankreas 8 mg, Kalbsthymus 4 mg, Pisum sativum 4 mg, Lens esculenta $4 \mathrm{mg}$ sowie Papayotin $2 \mathrm{mg}$, Mannit ad $100 \mathrm{mg}$

Dosierung u. Anwerdung: WOBE-MUGOS sollte grundsätzlich so hoch wie möglich dosiert werden Die nachfolgend angegebene Dosierung kann deshalb nur Anhaltspunkt sein $100 \mathrm{mg}$

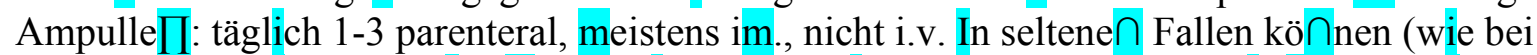
alien eiweißhaltigen Präparate $\cap$ ) allergische Sensationen (Rötungen, Juckreiz u a.), vereinzelt auch Anaphylaxien auftreten Derartige Reaktionen werden meist erst nach längeren Injektionsse $\pi$ en beobachtet. Es empfiehlt sich daher, nach 3-5 Injektionen auf Klistier-Tabletten umzustellen. - Zäpfchen: täglich 1-3 rektal, intravaginal. -Dragees dü $\pi$ ndarmlöslich: täglich 3-10 und mehr, unzerkaut schlucken. - Lutschdragees: täglich 3-6 langsam im Munde zergehen lassen

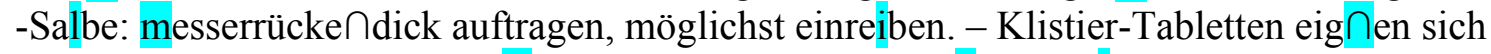
besonders für hone WOBE-MUGOS-Dosierungen über lä ggere Zeit. Näheres siehe Packungsbeilage

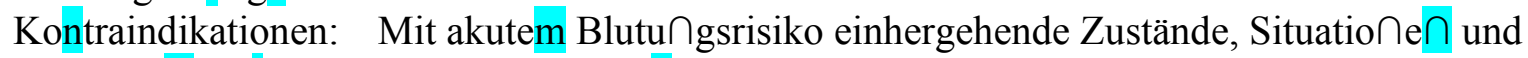
Erkranku gen, bei denen eine Steigerung der Fibrinolyse unerwünscht ist.

MUCOS Emulsionsgesellschaft mbH

Alpenstraße 29 8192 Geretsried 1 Tel. 08171/61061 